\title{
Special issue of 2017 India International Congress on Computational Intelligence
}

\author{
Suash Deb ${ }^{1,2} \cdot \mathrm{Ka}-$ Chun Wong ${ }^{3} \cdot$ Thomas Hanne $^{4}$ \\ Published online: 2 April 2020 \\ (C) Springer-Verlag London Ltd., part of Springer Nature 2020
}

Immense pleasure and a deep sense of gratitude we wish to present this special issue consisting of extended versions of a selected subset of papers presented at the twin conferences namely 5th International Conference on Computational and Business Intelligence (ISCBI17) and 1st International Conference on Intelligent Systems, Metaheuristics and Swarm Intelligence (ISMSI17), held in the year 2017, in Dubai and Hong Kong, respectively. A galaxy of researchers, academicians and industry professionals gathered to deliberate on various aspects covering the issues, challenges, theory as well as innovative applications of Soft Computing, Metaheuristics and other allied areas of computational intelligence systems at both conferences.

Following our long-standing tradition, after careful scrutiny we invited the authors of the best papers of both ISCBI17 and ISMSI17 conferences, as seleced by the team of experts as well as the guest editors to submit an extended version for possible publications at reputed SCIE indexed journal(s). For the year 2017, Neural Computing and Applications (NCAA) journal was chosen for that purpose. After undertaking an exhaustive reviewing of all of the submitted manuscripts, we have the pleasure of finally showcasing eight papers for this special issue-three from ISCBI17 and rest of that of ISMSI17.

Let us now proceed to provide a brief snapshot of those papers.

The study by Sengupta et al. reported the real-world applications of the reduction method of type-2 zigzag

Suash Deb

suash_deb@ieee.org

1 IT and Educational Consultant, Ranchi, Jharkhand, India

2 Decision Sciences and Modelling Program, Victoria University, Melbourne, Australia

3 City University of Hong Kong, Kowloon Tong, Hong Kong

4 University of Applied Sciences and Arts Northwestern Switzerland, Windisch, Switzerland uncertain variables. It has been compared with type- 2 triangular fuzzy variables. The results demonstrate that the proposed approach can solve the multi-objective solid transportation problem with robustness towards different credibility levels of profit, emission, source, demand, and conveyance in the context of carbon emission optimization.

Srivastava et al. have proposed a pattern-based image retrieval using Gray-Level Co-occurrence Matrix (GLCM) which is a very traditional technique in image texture analysis. The authors have re-examined the use of GLCM in image retrieval and provided refreshing insights. We conceive that it could be related to the convolution kernels commonly applied in deep convolutional neural network approaches.

On the other hand, Yuji Yoshida has studied the portfolio optimization problem with fuzzy random variables, accounting for coherent risk measures. The proposed approach not only can estimate randomness but also fuzziness as evaluated by lambda-mean functions and evaluation weights. Numerical examples are given to demonstrate the utility of the proposed approach with mathematical programming.

The study reported by Fakhr et al. has proposed the integration of Siamese-twin neural network hashing for unsupervised image retrieval. The authors have proposed two methods in the study. The first one is the design of Siamese-twin random projection neural network for image hashing; the second one is the bagging tree retrieval algorithm. The results on the COREL1K dataset and the CIFAR10 dataset support the effectiveness of the proposed methods.

Akbaripour et al. reported the real-world application to service composition and optimal selection in cloud manufacturing using a hybrid imperialist competitive and local search algorithm. The benchmark results based on online motorcycle production in the USA and four randomly generated large-scale instances suggested that the proposed approach can outperform the standalone local search 
method as well as the basic imperialist competitive algorithm in an efficient manner.

Interestingly, Fister Jr et al. have developed a particle swarm optimization framework for modeling preference times in triathlon. The authors recognized the heavy manual inspection time on preference time selection in triathlon. Therefore, they have proposed the framework for addressing the selection with practical experiments in different real-world scenarios with promising performance.

Simultaneously, Fister et al. have developed a different evolution method for post hoc analysis of athlete performance. In particular, they have proposed the use of heart rate data for the post hoc analysis. The proposed method has been tested on two case studies of running athletes, demonstrating its practical uses.

Last but not least, Chen et al. have proposed a convolutional neural network for Twitter comment sentiment analysis. Notably, the authors have manually annotated and created a novel dataset from Twitter comments. Extensive experiments have been conducted, demonstrating that the proposed convolutional neural network approach can outperform the existing methods. Visual analysis on the neural layers has also been conducted to reveal the reasons behind the good performance.
For the rapidly changing field of computational intelligence, there is no denying the fact that a number of fundamental issues remain to be addressed and many questions remain unanswered till now. Keeping that in mind, the guest editors had endeavored to focus on recent as well as novel progresses and hope that this special issue will further strengthen and provide more insight into future trends of various components of computational intelligence before it can flourish.

We take this opportunity of thanking all the authors who expanded their respective conference manuscripts as per the guidelines provided and submitted the same and showing great patience throughout the lengthy reviewing process. All the reviewers involved deserve our heartfelt gratitude for voluntarily spending time for studying the contents of the papers and sharing their findings with the guest editors. To conclude, we wish to acknowledge the help of the editor-in-chief of the NCAA journal for endorsing the special issue of the journal and also sharing his wisdom whenever solicited.

With our best wishes, renewed gratitude and warm personal regards to all.

Publisher's Note Springer Nature remains neutral with regard to jurisdictional claims in published maps and institutional affiliations. 